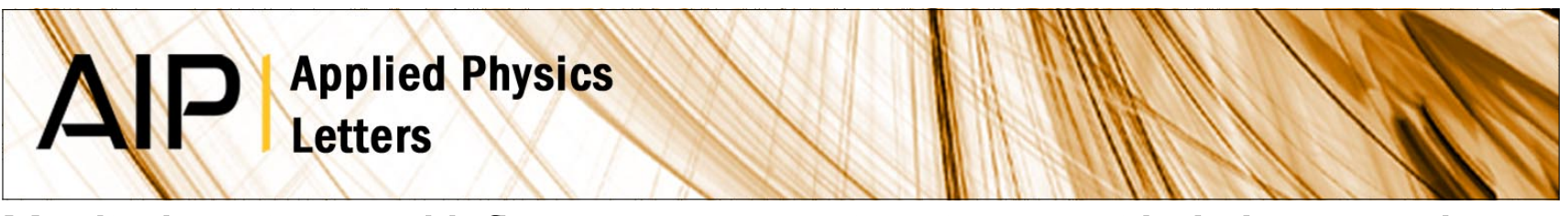

\title{
Monitoring structural influences on quantum transport in InAs nanowires
}

Robert Frielinghaus, Kilian Flöhr, Kamil Sladek, Thomas E. Weirich, Stefan Trellenkamp et al.

Citation: Appl. Phys. Lett. 101, 062104 (2012); doi: 10.1063/1.4742326

View online: http://dx.doi.org/10.1063/1.4742326

View Table of Contents: http://apl.aip.org/resource/1/APPLAB/v101/i6

Published by the American Institute of Physics.

Additional information on Appl. Phys. Lett.

Journal Homepage: http://apl.aip.org/

Journal Information: http://apl.aip.org/about/about_the_journal

Top downloads: http://apl.aip.org/features/most_downloaded

Information for Authors: http://apl.aip.org/authors

\section{ADVERTISEMENT}

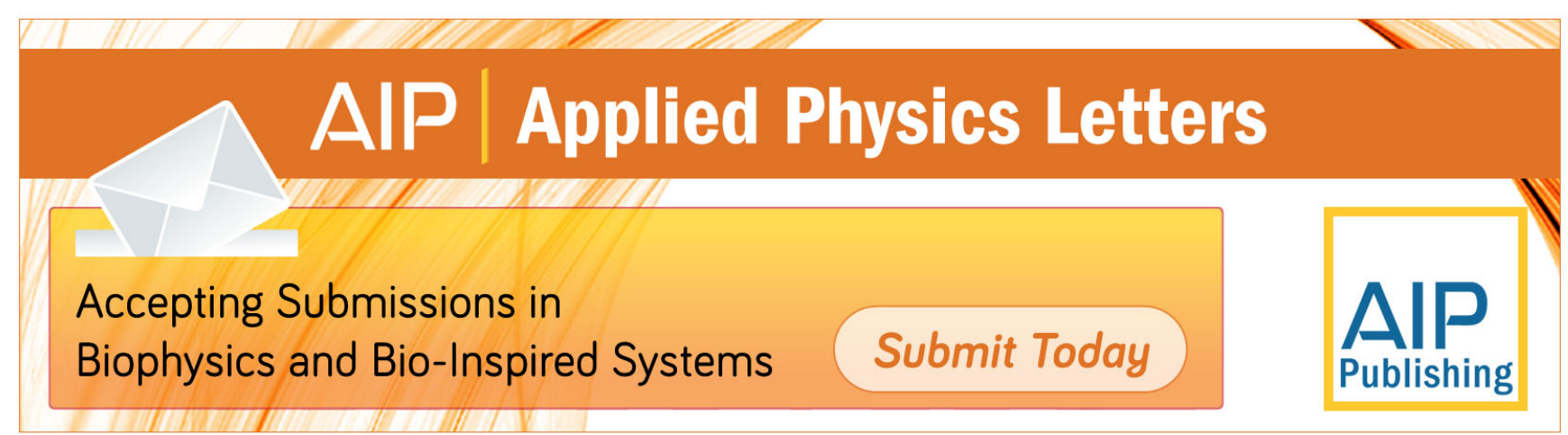




\title{
Monitoring structural influences on quantum transport in InAs nanowires
}

\author{
Robert Frielinghaus, ${ }^{1,2, a)}$ Kilian Flöhr, ${ }^{2,3}$ Kamil Sladek, $^{2,4}$ Thomas E. Weirich, ${ }^{2,5}$ \\ Stefan Trellenkamp, ${ }^{2,6}$ Hilde Hardtdegen, ${ }^{2,4}$ Thomas Schäpers, ${ }^{2,3,4}$ Claus M. Schneider, ${ }^{1,2}$ \\ and Carola Meyer ${ }^{1,2}$ \\ ${ }^{1}$ Peter Grünberg Institut 6, Forschungszentrum Jülich, 52425 Jülich, Germany \\ ${ }^{2}$ JARA-Fundamentals of Future Information Technologies \\ ${ }^{3}$ II. Physikalisches Institut, RWTH Aachen University, 52074 Aachen, Germany \\ ${ }^{4}$ Peter Grünberg Institut 9, Forschungszentrum Jülich, 52425 Jülich, Germany \\ ${ }^{5}$ Central Facility for Electron Microscopy GFE, RWTH Aachen University, 52074 Aachen, Germany \\ ${ }^{6}$ Peter Grünberg Institut 8-PT, Forschungszentrum Jülich, 52425 Jülich, Germany
}

(Received 21 May 2012; accepted 19 July 2012; published online 8 August 2012)

\begin{abstract}
A sample design that allows for quantum transport and transmission electron microscopy (TEM) on individual suspended nanostructures is used to investigate moderately $n$-type doped InAs nanowires (NWs). The nanowires were grown by metal organic vapor phase epitaxy. Universal conductance fluctuations in the nanowires are investigated at temperatures down to $0.35 \mathrm{~K}$. These fluctuations show two different temperature dependences. The very same nanowire segments investigated in transport are subsequently analyzed by TEM revealing crystal phase mixing. However, we find no correspondence between the atomic structure of the wires and the temperature dependences of the conductance fluctuations. @ 2012 American Institute of Physics. [http://dx.doi.org/10.1063/1.4742326]
\end{abstract}

Self-assembled semiconductor nanowires (NWs) receive a considerable interest as possible candidates for future nanoelectronic devices. ${ }^{1-3}$ Here, some III/V compounds with a low bandgap as, e.g., InAs, InN, or InSb exhibit a surface accumulation layer that leads to Schottky-barrier free contacts what in turn facilitates integration. ${ }^{4-6}$ Besides this application-driven research, various fundamental quantum effects can be studied in these material systems. Confinement and interference effects start to dominate the electronic transport at cryogenic temperatures, and single-electron tunneling or phase coherent transport can be observed. ${ }^{7-9}$

However, there are two factors inherent in the device geometry that greatly influence standard transport experiments and result in an experimental fingerprint for each nanowire. First, the high aspect ratio and enhanced surface conduction lead to an increased sensitivity to the environment. This can be used, e.g., for gas sensing ${ }^{10}$ but may also be obstructive, since the substrate can affect the transport considerably. Second, an usually unknown defect distribution individual for each nanowire is used to explain many quantum interference effects and transport phenomena. . $^{8,112}$

In this Letter, we use a sample design where individual InAs nanowires are suspended across holes in a $\mathrm{Si}_{3} \mathrm{~N}_{4}$ transmission electron microscopy (TEM) membrane. The NWs are subsequently contacted and universal conductance fluctuations (UCFs) are measured at cryogenic temperatures. We find highly reproducible fluctuation patterns and clear temperature dependences as we do not have any substrate influence in our suspended geometry. Two distinct temperature dependences of the phase coherence length are observed in a set of four wires. We correlate these transport data with the atomic structure of the NWs obtained in a successive TEM measurement on the very same wire section.

This approach has two advantages. In the suspended geometry, all substrate effects on the transport can be excluded.

${ }^{a)}$ Electronic mail: r.frielinghaus@fz-juelich.de.
Although previous measurements ${ }^{6}$ found a similar crystal structure in a set of NWs, there is still a certain spread. A wire-to-wire comparison of these data may reveal a connection that could remain unnoticed otherwise.

InAs nanowires were grown on a GaAs (111)B substrate by selective-area metal-organic vapour phase epitaxy (SAMOVPE) and moderately $n$-doped by $\mathrm{Si}$ incorporation. ${ }^{13} \mathrm{~A}$ pressure ratio of dopant versus group III precursor of $p\left(\mathrm{Si}_{2} \mathrm{H}_{6}\right) / p(\mathrm{TMIn})=7.5 \cdot 10^{-3}$ was chosen (doping factor 100 in Ref. 6). The resulting NWs have a diameter of $110 \mathrm{~nm}$ and a length of about $4 \mu \mathrm{m}$. A room-temperature resistivity of $\rho \approx 200 \mu \Omega \cdot \mathrm{m}$ and a carrier concentration of $n \approx 1 \cdot 10^{18} \mathrm{~cm}^{-3}$ were obtained from previous measurements ${ }^{6}$ on NWs of similar growth conditions. We estimate the contact resistances with these values and the results from the multiterminal wires.

Individual as-grown NWs were mechanically removed from the GaAs growth substrate with an In needle and selectively placed across holes of a TEM membrane. ${ }^{14}$ All NWs were chosen from within an area of about only $20 \times 20 \mu \mathrm{m}^{2}$ edge length, so their individual growth conditions were expected to be very similar. The TEM membrane was a perforated DuraSiN DTM-25232 with a coordinate system previously fabricated by electron beam lithography consisting of $\mathrm{Ti} / \mathrm{Pt}$ markers $(5 \mathrm{~nm} / 60 \mathrm{~nm})$. Individual Ti/Au $(10 \mathrm{~nm} /$ $120 \mathrm{~nm}$ ) contacts to the nanowires were again lithographically defined. The NWs were exposed to an oxygen ion beam and to an $\mathrm{Ar}^{+}$plasma prior to metal evaporation to ensure a good contact transparency. The lithography on the membranes is described in more detail elsewhere. ${ }^{15}$ TEM images of the samples were acquired in an FEI Tecnai F20 at an acceleration voltage of $200 \mathrm{kV}$ after the transport measurements.

We prepared four samples in this manner, each having a suspended length of around $1 \mu \mathrm{m}$. A scanning electron micrograph of one of the NWs (wire $A$ ) is depicted in Fig. 1. The other devices are built in a three- (wire $D$ ) and two-terminal configuration (wires $B$ and $C$ ). The transport 


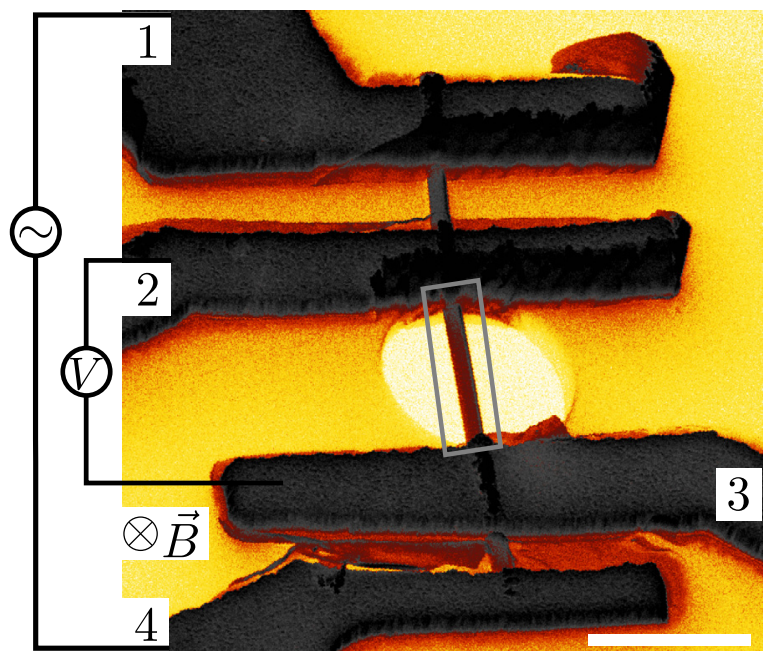

FIG. 1. Scanning electron micrograph of wire $A$ with a schematics of the configuration used in the transport measurements. Scale bar is $1 \mu \mathrm{m}$. The color scale of the secondary electron yield is inverted for clarity. A TEM image of the marked middle section is displayed in Fig. 3.

measurements were conducted in a He-3 cryostat at temperatures between $0.35 \mathrm{~K}$ and $30 \mathrm{~K}$. The resistance was measured using a standard lock-in technique with an ac bias of $20 \mathrm{nA}$ (wire $B$ ), $30 \mathrm{nA}$ (wires $C$ and $D$ ), and $50 \mathrm{nA}$ (wire $A$ ). A contact scheme for the four-terminal case is indicated in Fig. 1. A magnetic field up to $7 \mathrm{~T}$ was applied perpendicular to the wire axis.

The magnetoconductance fluctuates as a function of the magnetic field at low temperatures as can be seen in Fig. 2(a). To quantify the fluctuation $\delta_{G}=G-G_{0}$, we subtract a slowly varying background $G_{0}$ from the wire conductance, which is in turn determined from the measured wire resistance after subtracting any contact resistances. An exemplary pattern is depicted in the left part of Fig. 2(a). The pattern reproduces itself with a decreasing root mean square of the amplitude, $\sigma_{\delta_{G}}$, upon increasing the temperature (right part of Fig. 2(a)). This variation in magnetoconductance can be attributed to UCFs. ${ }^{16,17}$ The amplitude stays constant up to $1 \mathrm{~K}$ and decreases exponentially as $T^{-0.70 \pm 0.04}$ for $T \geq 2 \mathrm{~K}$ for all wires (Fig. 2(b)). This can be explained with the phase-coherence length $l_{\varphi}$ being comparable or longer than the contact separation at $T \leq 1 \mathrm{~K}$ as discussed below. For $T \geq 2 \mathrm{~K}$, two effects divide the wire into several smaller phase-coherent sections. First, small energy transfer electron-electron scattering (Nyquist mechanism) dephases the electrons. ${ }^{18}$ Second, thermal broadening leads to an interference of several states contributing to the transport. The latter can be quantified by the thermal length, $l_{T}=\sqrt{\hbar \mathcal{D} / k_{B} T}$, with $\mathcal{D}$ being the diffusion constant.

The phase-coherence length $l_{\varphi}$ can be determined from the correlation field $B_{C}$. The latter is defined via the autocorrelation function, $f(\Delta B)=\left\langle\delta_{G}(B+\Delta B) \delta_{G}(B)\right\rangle_{B}$, with $f\left(B_{C}\right):=\frac{1}{2} f(0)$. Here, $\langle\ldots\rangle_{B}$ denotes the average over the magnetic field. A dependence

$$
l_{\varphi}=\gamma \Phi_{0} /\left(d B_{C}\right)
$$

is predicted for channels with $d<l_{\varphi}$ as in our case. ${ }^{17,19}$ Here, $d$ is the channel width (the diameter, in our samples),

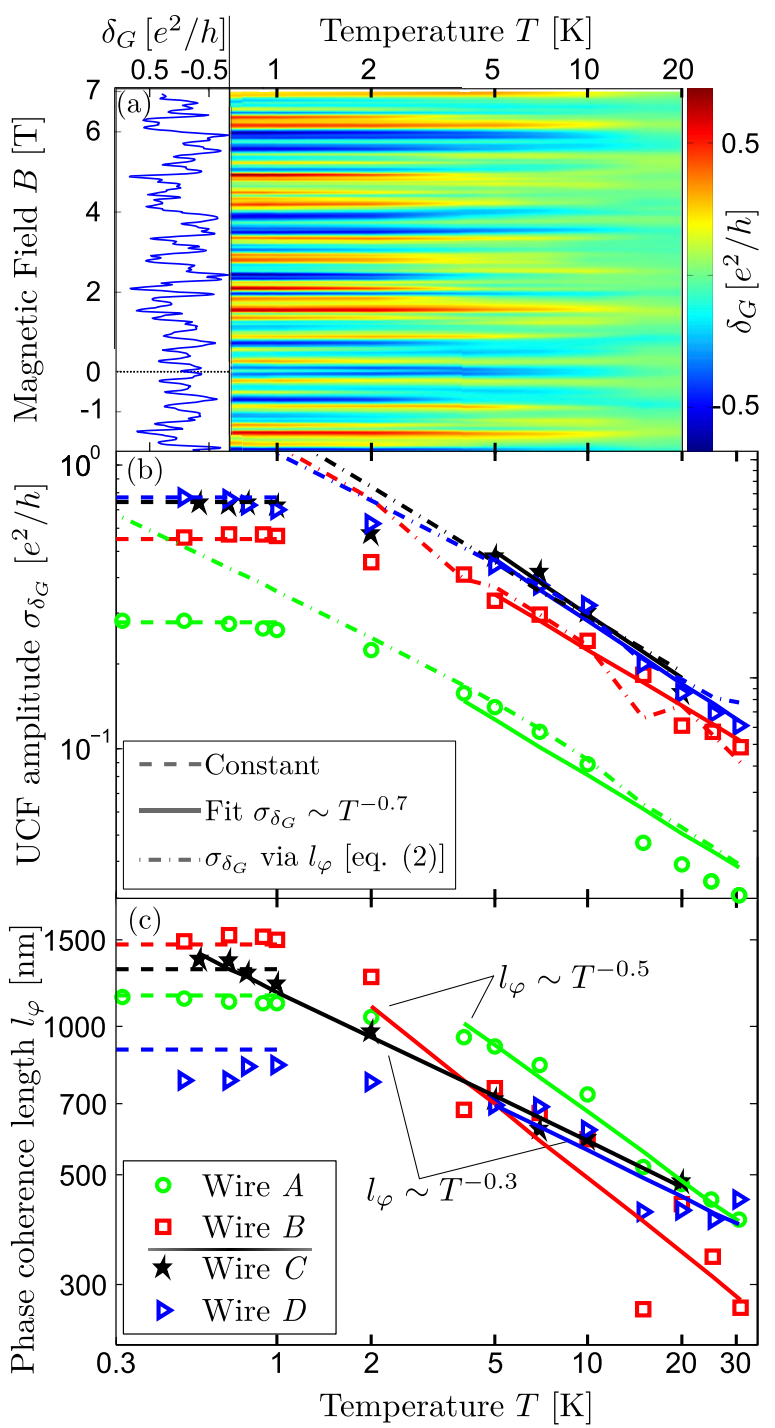

FIG. 2. (a) Universal conductance fluctuation pattern $\delta_{G}$ of wire $A$ in units of $e^{2} / h$ as a function of magnetic field and temperature. The curve on the left side shows the conductance fluctuations at $0.35 \mathrm{~K}$. (b) UCF amplitude $\sigma_{\delta_{G}}$ vs $T$ of the investigated suspended wires. The dashed lines indicate the maximum constant amplitude when the entire wire is phase-coherent. The solid lines represent the fitted temperature dependences above $3 \mathrm{~K}$. The dash-dotted line is the UCF amplitude calculated from the phase-coherence length $l_{\varphi}$ using Eq. (2). (c) $l_{\varphi}$ vs $T$ determined from the correlation field $B_{C}$. The solid lines are the fitted exponential temperature dependences. The dashed horizontal lines indicate the contact separation length.

$\Phi_{0}=h / e$ the magnetic flux quantum, and $\gamma$ a proportionality constant between 0.42 and 0.95 for $l_{T} \gg l_{\varphi}$ and $l_{T} \ll l_{\varphi}$, respectively.

The phase-coherence length determined from $B_{C}$ is displayed in the Fig. 2(c). We observe a similar behavior as with the UCF amplitude, i.e., a saturation below $1 \mathrm{~K}$ and an exponential decrease above $3 \mathrm{~K}$ for three wires. Only $l_{\varphi}$ of wire $C$ starts decreasing at lower temperatures. We find a quantitative agreement of $\max \left(l_{\varphi}\right)$ with the contact separation length $L$ for all wires if we assume $\gamma \approx 2$. This agreement confirms our above assumption that the entire wire sections between the contacts are phase-coherent for $T \leq 1 \mathrm{~K}$.

The determined value for $\gamma$ is about two times larger than predicted, since it is at least $l_{T} \lesssim 0.2 l_{\varphi}$ for all wires and temperatures. The theories used in this Letter have been 


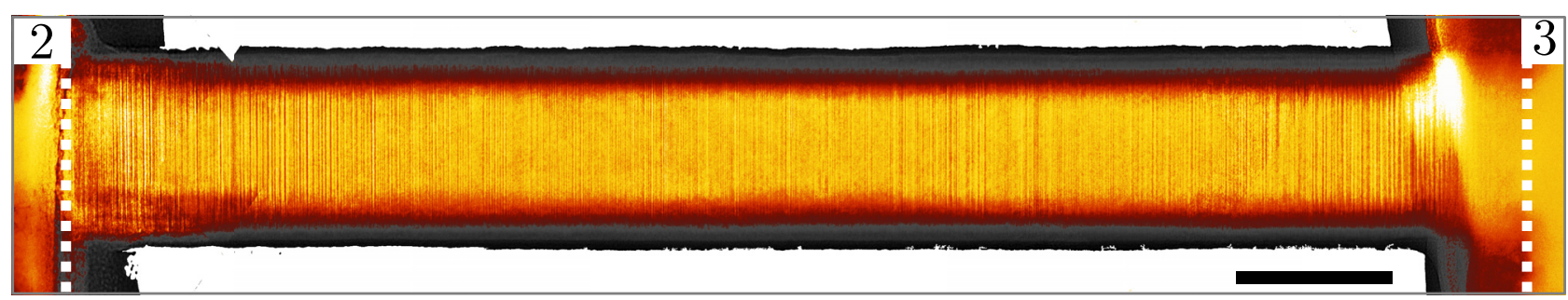

FIG. 3. Low-magnification TEM image of the middle section of wire $A$. Scale bar is $100 \mathrm{~nm}$. The image is stitched from 18 individual micrographs. The dashed lines mark the extremities of contacts 2 and 3 as indicated in Fig. 1.

developed for wires made from two-dimensional planar electron gases, i.a., they do not account for flux cancellation effects owing to the cylindrical shape of our NWs and recently reported in InAs NWs grown by molecular beam epitaxy (MBE). ${ }^{9}$ This flux cancellation leads to an overestimation of $B_{C}$, which in turn increases the value of $\gamma$. A value of $\gamma \approx 1.4$ was obtained for the MBE-grown NWs, which is close to our finding $\gamma \approx 2$ for MOVPE-grown samples. The difference can be explained by the fact that the former were measured in the $l_{T} \gg l_{\varphi}$ regime where $\gamma$ is expected to be smaller. ${ }^{17,19}$

We observe two distinct dephasing rates of $l_{\varphi}$ upon increasing the temperature. We obtain $l_{\varphi} \sim T^{-0.5}$ for wires $A$ and $B$ while $l_{\varphi}$ in wires $C$ and $D$ decays with $T^{-0.3}$. In contrast, the decrease of the UCF amplitude is predicted to depend mainly on $l_{T}$ in the regime of $l_{T} \ll l_{\varphi} \ll L$ as it is the case for $T \geq 3 \mathrm{~K}$. It is ${ }^{17,19}$

$$
\sigma_{\delta_{G}}=\beta \frac{e^{2}}{h} \frac{l_{T}}{L} \sqrt{\frac{l_{\varphi}}{L}}
$$

with $\beta=\sqrt{8 \pi / 3}$. Again our data fits nicely to the theoretical prediction as shown in Fig. 2(b). However, we have to modify $\beta$ similar to $\gamma$ by a factor between 0.5 (wire $A$ ) and 2 (wire $B$ ) to obtain a quantitative agreement. The reason for the distinct prefactors is likely a slight deviation of the real contact resistances to the estimated values. A geometrical effect as in the discussion of $l_{\varphi}$ above may lead to an additional constant deviation of $\beta$.

We can estimate the error on the various fitting parameters like $\gamma$ and the decay exponents to be around $10 \%$ from a repetitive measurement after one thermal cycle to room temperature.

The origin of the different temperature dependences is unclear at this point. We can neglect all detrimental substrate effects due to the suspended geometry of our samples. Thus, this origin can only lie within the structure of the wires themselves. The devices show no difference on the length scales accessible in scanning electron microscopy. Therefore, we take advantage of our sample design and use TEM to investigate if the atomic structure of the wires has an influence on the UCFs and on the different dephasing rates of $l_{\varphi}$. These measurements were performed as a last step to avoid any influence of the transport properties by the defects possibly induced from the electron beam irradiation. A lowmagnification image stitched from 18 individual micrographs of the suspended part of wire $A$ is shown in Fig. 3. The edges of the inner contacts can be seen at the image borders. The vertical fringes are due to stacking faults between different crystal structures (see below). All four wires show no systematic differences in terms of diameter, contact separation, suspended respectively supported length, disorder, and dirt particles in this magnification.

We observe individual atomic planes and identify wurtzite and zinc blende crystal phases and an amorphous surface layer of 3-4 nm thickness upon increasing the magnification. A sample plot is shown in Fig. 4(a). The different crystal structures have a conduction band offset between $23 \mathrm{meV}$ (Ref. 20) and $86 \mathrm{meV}$ (Ref. 21) and are discussed as the origin of various transport phenomena: Conductivity and transport activation were shown to depend on the degree of mixing. ${ }^{11,12,22}$ Wurtzite areas may act as tunnel barriers for quantum dots. ${ }^{8,23}$

An influence of the crystal phase mixing on the UCFs seems reasonable because the Nyquist dephasing that is expected to be dominant in epitaxial semiconductor NWs is sensitive to the density of electrons ${ }^{18}$ via the number of states to scatter from and to. Additionally, the phase
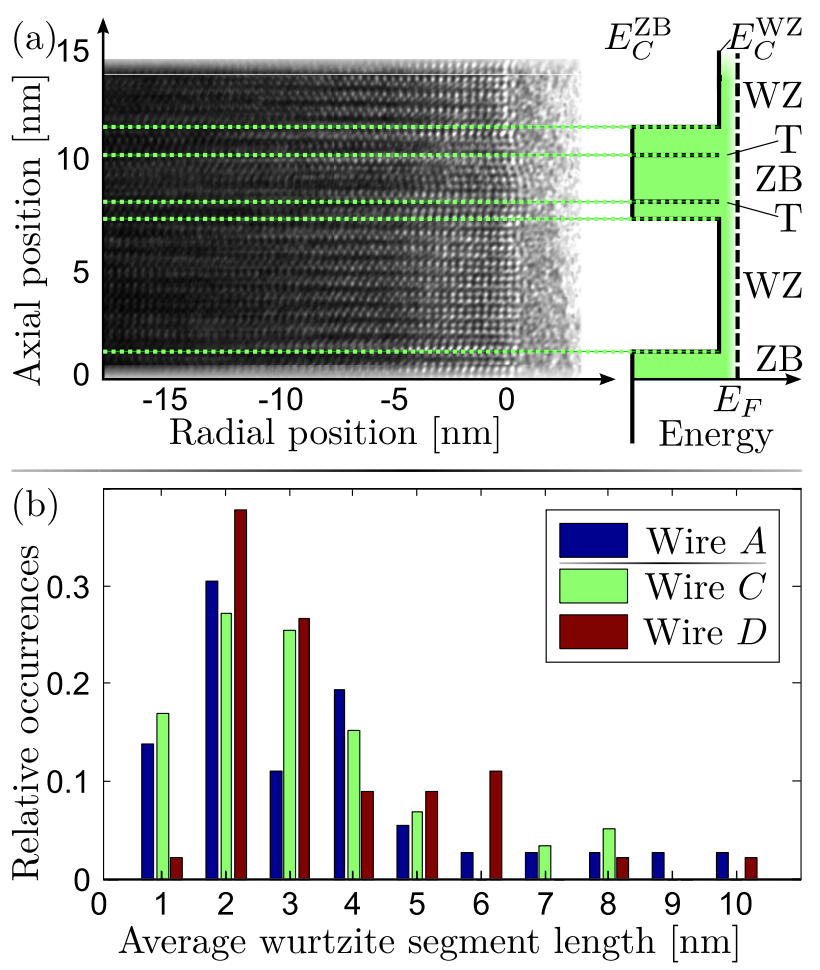

FIG. 4. (a) Example high-resolution TEM image of wire $A$ along with a schematics of the conduction band profile $E_{C}$ of the wurtzite (WZ) and zinc blende (ZB) crystal phases with twinning planes (T). $E_{F}$ marks the Fermi energy. (b) Distribution of the axial wurtzite segment lengths of the investigated nanowires. 
coherence length depends on the diffusion constant $\mathcal{D}$ that in turn reflects the disorder in the crystal. This disorder has two main contributions, namely, the crystal phase boundaries and point defects, e.g., from the dopants.

In previous experiments, it was not possible to correlate the crystal structure with the transport properties of the very same wire. Therefore, statistical fluctuations of the wire properties, i.e., atomic structure and doping, which typically occur in a sample, could distort the interpretation of the transport data. Here, we present a direct mapping of the crystal phases along the current flow (see right part of Fig. 4(a)) and correlate the phase mixing with the transport data of the very same wire.

We evaluate the typical wurtzite segment length and, therefore, the potential fluctuation periodicity in Fig. 4(b). All distributions exhibit an average segment length of $3 \mathrm{~nm}$ and a similar profile. The same holds for the zinc blende segments (not shown). Thus, the fluctuations in the crystal phase cannot account for the distinct transport behaviours. We can also rule out the surface layer that consists of mainly indium oxide $^{7}$ and some remnants of the PMMA resist as it does not vary between the individual wires.

We thus have to account the transport property variations to differences that we cannot extract from the TEM measurements. One possibility is the local dopant and atomic defect distribution that affects the diffusion constant $\mathcal{D}$. Another candidate is the strain resulting, e.g., from the suspended geometry, the clamping by the contacts, and the different thermal expansion coefficients of the InAs and the $\mathrm{Si}_{3} \mathrm{~N}_{4}$.

In summary, we have investigated freely suspended InAs nanowires with a sample design that offers two major advantages to standard transport experiments. First, with the suspended geometry, no substrate interaction can affect the low-temperature transport experiments. Second, it is possible to subsequently measure the crystal structure of the very same nanowires by transmission electron microscopy and to thus correlate transport and crystalline properties. The NWs show very clear universal conductance fluctuations unperturbed by any substrate influence at cryogenic temperatures. We could extract, i.a., the phase coherence length that showed significantly different temperature dependences from these UCFs. All nanowires exhibit crystal phase mixing between zinc blende and wurtzite structure in the TEM measurements. This phase mixing cannot account for the different transport properties, since the NWs show no systematic differences. We will further investigate the influence of strain and dopants on the temperature dependence of the phase coherence length in future measurements. This can be done by studying wires with different crystal structures and doping in transport experiments. Electron holography can be used to investigate the distribution of the electronic potential within a wire. The sample design presented here can be used to correlate the results obtained with these different methods.

We thank Martin Schuck and Herbert Kertz for measurement support, Beata Kardynal for fruitful discussions, and Falk Dorn for recording the TEM images. We also acknowledge financial support from the DFG Forschergruppe 912 and from the RWTH Aachen via an ERS seed fund.

${ }^{1}$ L. Samuelson, C. Thelander, M. Börk, M. Borgström, K. Deppert, K. Dick, A. Hansen, T. Mårtensson, N. Panev, A. Persson, W. Seifert, N. Sköld, M. Larsson, and L. Wallenberg, Physica E 25, 313 (2004).

${ }^{2}$ Y. Li, F. Qian, J. Xiang, and C. M. Lieber, Mater. Today 9, 18 (2006).

${ }^{3}$ P. Yang, R. Yan, and M. Fardy, Nano Lett. 10, 1529 (2010).

${ }^{4}$ D. C. Tsui, Phys. Rev. Lett. 24, 303 (1970).

${ }^{5}$ M. Noguchi, K. Hirakawa, and T. Ikoma, Phys. Rev. Lett. 66, 2243 (1991).

${ }^{6}$ S. Wirths, K. Weis, A. Winden, K. Sladek, C. Volk, S. Alagha, T. E. Weirich, M. von der Ahe, H. Hardtdegen, H. Lüth, N. Demarina, D. Grützmacher, and T. Schäpers, J. Appl. Phys. 110, 053709 (2011).

${ }^{7}$ I. Shorubalko, A. Pfund, R. Leturcq, M. T. Borgström, F. Gramm, E. Müller, E. Gini, and K. Ensslin, Nanotechnology 18, 044014 (2007).

${ }^{8}$ M. D. Schroer and J. R. Petta, Nano Lett. 10, 1618 (2010).

${ }^{9}$ C. Blömers, M. I. Lepsa, M. Luysberg, D. Grützmacher, H. Lüth, and T. Schäpers, Nano Lett. 11, 3550 (2011).

${ }^{10}$ J. Du, D. Liang, H. Tang, and X. P. Gao, Nano Lett. 9, 4348 (2009).

${ }^{11}$ J. Wallentin, M. Ek, L. R. Wallenberg, L. Samuelson, and M. T. Borgström, Nano Lett. 12, 151 (2012).

${ }^{12}$ C. Thelander, P. Caroff, S. Plissard, A. W. Dey, and K. A. Dick, Nano Lett. 11, 2424 (2011).

${ }^{13}$ K. Sladek, A. Penz, K. Weis, S. Wirths, C. Volk, S. Alagha, M. Akabori, S. Lenk, M. Luysberg, H. Lüth, H. Hardtdegen, T. Schäpers, and D. Grützmacher, MRS Proc. 1258, 2 (2010).

${ }^{14}$ K. Flöhr, M. Liebmann, K. Sladek, H. Y. Günel, R. Frielinghaus, F. Haas, C. Meyer, H. Hardtdegen, T. Schäpers, D. Grützmacher, and M. Morgenstern, Rev. Sci. Instrum. 82, 113705 (2011).

${ }^{15}$ R. Frielinghaus, K. Goß, S. Trellenkamp, L. Houben, C. M. Schneider, and C. Meyer, Phys. status Solidi B 248, 2660 (2011).

${ }^{16}$ B. Al'tshuler, Pis'ma Zh. Eksp. Teo. Fiz. 41, 530 (1985) [JETP Lett. 41, 648 (1985)].

${ }^{17}$ P. A. Lee, A. D. Stone, and H. Fukuyama, Phys. Rev. B 35, 1039 (1987).

${ }^{18}$ B. L. Al'tshuler, A. G. Aronov, and D. E. Khmelnitsky, J. Phys. C 15, 7367 (1982).

${ }^{19}$ C. W. J. Beenakker and H. van Houten, Phys. Rev. B 37, 6544 (1988).

${ }^{20}$ A. De and C. E. Pryor, Phys. Rev. B 81, 155210 (2010).

${ }^{21}$ M. Murayama and T. Nakayama, Phys. Rev. B 49, 4710 (1994).

${ }^{22}$ S. A. Dayeh, D. Susac, K. L. Kavanagh, E. T. Yu, and D. Wang, Adv. Funct. Mater. 19, 2102 (2009).

${ }^{23}$ K. A. Dick, C. Thelander, L. Samuelson, and P. Caroff, Nano Lett. 10, 3494 (2010). 